
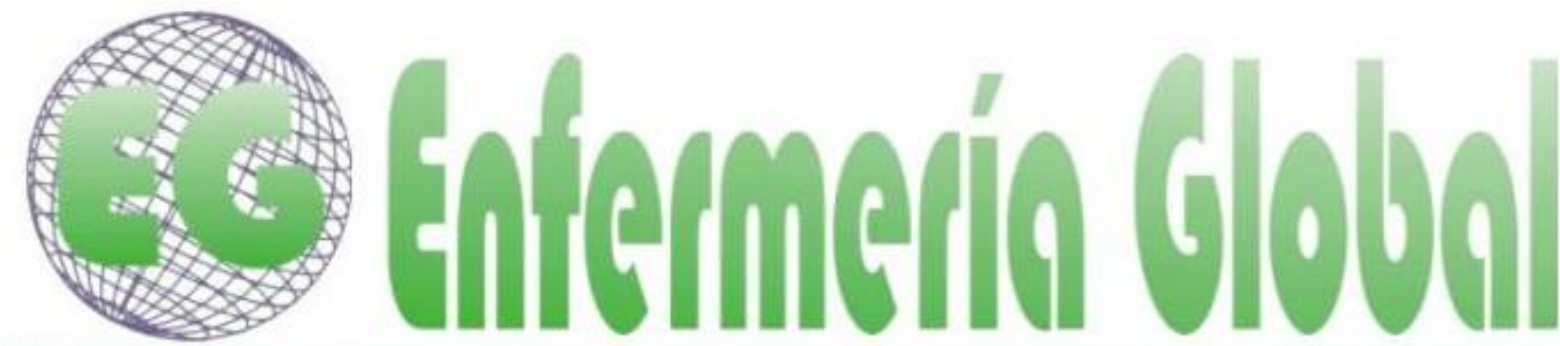

\title{
Expectativas de parto de las gestantes de La Ribera: una aproximación cualitativa
}

Childbirth expectations of La Ribera pregnant women: a qualitative approach

\section{Francisca Camacho Morell ${ }^{1}$ \\ $\mathrm{M}^{\mathrm{a}}$ - José Romero Martín ${ }^{2}$}

1 Enfermera especialista en Obstetricia y Ginecología (Matrona). Doctoranda del programa de Doctorado en Enfermería clínica y comunitaria de la Universidad de Valencia. Matrona asistencial en el paritorio del Hospital Universitario de La Ribera. Alzira. Valencia. España.

2 Enfermera especialista en Obstetricia y Ginecología (Matrona). Matrona asistencial en el paritorio del Hospital Universitario de La Ribera. Alzira. Valencia. España

\section{E-mail: francisca.camacho@uv.es}

\section{http://dx.doi.org/10.6018/eglobal.16.4.276061}

Recibido: $24 / 11 / 2016$

Aceptado:07/04/2017

\section{RESUMEN:}

Objetivo: El cambio de modelo asistencial en la atención al parto normal, el interés por parte de las autoridades sanitarias en que se exploren las expectativas de parto de las gestantes, y su especificidad cultural y social, hacen que el estudio de tales expectativas sea pertinente en este momento. El objetivo del presente estudio es conocer cuáles son las expectativas de parto de una muestra de gestantes españolas y estudiar las diferencias entre las expectativas de las gestantes primíparas y multíparas.

Metodología: Estudio cualitativo basado en una pregunta de respuesta libre sobre expectativas de parto. Se recogieron datos a gestantes de tercer trimestre del área de salud de La Ribera (Alzira, Valencia) durante 2014-2015. Se analizaron los datos mediante análisis del contenido.

Resultados: Muestra de 213 gestantes cuyas principales expectativas de parto fueron tener un parto rápido, con buen resultado obstétrico, sin dolor y con apoyo de los profesionales. Las expectativas relacionadas con los protocolos de atención al parto normal supusieron un $5.2 \%$ del total de las respuestas. No se encontraron diferencias estadísticamente significativas entre gestantes primíparas y multíparas.

Conclusiones: El conocimiento de las expectativas resulta importante, ya que las gestantes miden su satisfacción con el parto en base al cumplimiento de tales expectativas. Ayudarlas a desarrollar expectativas realistas aumentará su satisfacción. Las matronas tienen un importante papel a través de la formación que imparten (educación maternal) y del apoyo en el momento del parto (fundamental para que las gestantes se sientan protagonistas del mismo).

Palabras clave: expectativas de parto; investigación cualitativa; embarazo; matronas.

\section{ABSTRACT:}

Objective: Change of healthcare model in normal childbirth, health authorities interest in exploring childbirth expectations of pregnant women, and their cultural and social specificity make relevant the study of these expectations at this moment. The aim is to know what are the childbirth expectations 
from a sample of Spanish pregnant women and to study the differences between primiparous and multiparous pregnant women expectations.

Methodology: Qualitative study based on an open-ended question about childbirth expectations. Data were collected during 2014-2015 to third trimester pregnant women in La Ribera health area (Alzira, Valencia). Data were analyzed using content analysis.

Results: Sample of 213 pregnant women whose main expectations were to have a fast delivery, with good obstetric outcome, painlessly and with professional support. Expectations regarding normal childbirth protocols accounted for $5.2 \%$ of total responses. No statistically significant differences between primiparous and multiparous pregnant women were found.

Conclusions: Knowledge about childbirth expectations is highly relevant because pregnant women measure their satisfaction with childbirth through the fulfillment of these expectations. Helping them to develop realistic expectations will increase their satisfaction. Midwives play an important role through the training they give (maternal education) and through the support during delivery (this is essential for the pregnant women in order to feel themselves protagonist of their delivery).

Keywords: childbirth expectations; qualitative research; pregnancy; nurse midwives.

\section{INTRODUCCIÓN}

El parto es un evento complejo en la vida de cualquier mujer, caracterizado por rápidas transiciones biológicas, sociales y emocionales, donde influyen factores contextuales, políticos y sobre todo culturales ${ }^{(1)}$. Muchas mujeres experimentan la experiencia de parto como un momento crítico de auto-afirmación y un elemento central para el bienestar psicológico materno ${ }^{(2)}$. Una experiencia de parto negativa puede conducir a sentimientos de frustración y falta de control, y afectar a la decisión de la mujer acerca de una futura maternidad ${ }^{(3-4)}$.

Formarse expectativas sobre los momentos importantes de la vida puede ayudar a prepararse mental o psíquicamente para dicho momento ${ }^{(5)}$. Las mujeres utilizan tales expectativas para evaluar su experiencia de parto ${ }^{(3,6)}$. Por lo tanto, la satisfacción de la mujer con esa experiencia depende, en gran medida, de su concordancia con las expectativas previas ${ }^{(4,7-9)}$. Además, tales expectativas influyen en la respuesta y posterior adaptación de las mujeres a la maternidad ${ }^{(4,10)}$. La discordancia entre expectativas y experiencia de parto puede incluso llevar a las mujeres a dañar su confianza como madre y jugar un papel en el riesgo de depresión postparto ${ }^{(5)}$. Las gestantes se acercan al parto con unas expectativas determinadas y detalladas ${ }^{(3,8)}$, que pueden diferir significativamente de una mujer a otra y que se desarrollan con el paso del tiempo ${ }^{(10)}$. Además, dependen de condicionantes sociales que influyen en el sentido de decidir cuál es el comportamiento adecuado durante el parto y, debido a esta especificidad cultural y social, es difícil generalizarlas ${ }^{(3)}$.

El concepto de expectativas de parto está muy extendido en la literatura. Sin embargo, no hay consenso en cuanto a su definición ${ }^{(11)}$. Según Ayers ${ }^{(12)}$, la expectativa de parto es una construcción compleja y multidimensional que implica muchos aspectos del parto, y en este contexto cabría definirla, en relación al parto, como los "juicios y creencias sobre el futuro que pueden estar influidas por situaciones pasadas" ${ }^{\prime(13)}$.

Una gran parte de las investigaciones en este campo, ya desde el siglo pasado, se han centrado en el estudio de la relación entre expectativas y experiencias de parto $^{(3,8,14-16)}$ o en el análisis de las experiencias de parto en sí( ${ }^{6,17)}$. En algunos casos, la exploración de las expectativas se ha realizado en un momento posterior al parto, desde 48-72 horas tras el parto hasta varios meses, e incluso años después del mismo $^{(3,7-8,16,18)}$. No obstante, lo más habitual ha sido explorar las expectativas 
durante el tercer trimestre de embarazo ${ }^{(5,8-10,19-22)}$, momento en el que ya se han desarrollado, debido a la cercanía del parto, pero todavía no se tiene una experiencia del mismo.

En cuanto a la relación entre expectativas de parto y otras variables, destaca el análisis de las diferencias entre las mujeres que han parido anteriormente y aquellas que no tienen ninguna experiencia previa de parto. Según Peñacoba ${ }^{(19)}$, existe un amplio conocimiento de las expectativas de las mujeres que no tienen hijos, pero existen menos estudios que hayan explorado, por un lado, las expectativas de aquellas mujeres que han parido con anterioridad y, por otro, los que exploran la diferencia entre ambos colectivos. Así, algunos estudios encuentran que las expectativas de parto son más positivas en el caso de las multíparas que para las primíparas $^{(7,23)}$.

Respecto del tipo de estudio realizado, los de carácter cuantitativo se han basado en diferentes instrumentos de medida, como el Chilbirth Expectations Questionnaire (CEQ), el Wijma Delivery Expectancy/Experience Questionnaire (W-DEQ) o el Expectations of the Childbirth Experience (ECBE) ${ }^{(9,14)}$. Por su parte, los estudios de carácter cualitativo se basan en aspectos como el análisis del contenido ${ }^{(7,20)}$, la aproximación fenomenológica ${ }^{(8)}$, la comparación constante ${ }^{(3,18)}$ y los grupos focales ${ }^{(16-}$ 17). Los estudios cualitativos son particularmente adecuados para estudiar fenómenos complejos o procesos que son poco conocidos. Entre las ventajas de la aproximación cualitativa se encuentran, por un lado, poder analizar un conjunto de datos sin las presunciones o teorías generadas de antemano ${ }^{(24)} \mathrm{y}$, por otro lado, ser la metodología más apropiada para determinar los sentimientos, interacciones, percepciones y comportamientos individuales ${ }^{(25)}$.

En los últimos años se está produciendo un cambio en el sistema sanitario, desde una aproximación biomédica a otra más psicosocial, que tiene en cuenta las creencias y emociones de las mujeres $^{(19)}$ y reivindica un empoderamiento de estas ${ }^{(26)}$. En este contexto de cambio, el estudio de las expectativas de parto cobra un nuevo sentido. Desde diferentes instituciones se hace referencia a la importancia de que los profesionales conozcan las expectativas de cada mujer con respecto al proceso de parto, así como la importancia de investigar formas efectivas en que los profesionales de la salud pueden apoyar a las gestantes para tomar decisiones informadas durante el parto ${ }^{(27)}$. En esta misma línea, diferentes estudios enfatizan la importancia de explorar las expectativas y demandas sanitarias de las usuarias para incluirlas en la oferta del sistema sanitario y hacen hincapié en el insuficiente conocimiento de las expectativas de las usuarias con relación a la humanización de la asistencia al parto $^{(21)}$.

La bibliografía referida a España pone de relieve que no disponemos de conocimientos suficientes sobre las expectativas de parto de las gestantes. Esta laguna de conocimiento es aún más importante en el caso de estudios realizados con metodología cualitativa. Existen estudios cualitativos que analizan la experiencia de parto mediante la técnica del grupo focal en nuestro país ${ }^{(16-17)}$, pero no las expectativas previas al parto. Así pues, nos encontramos con 3 factores importantes:

- el cambio de modelo asistencial en la atención al parto normal

- la especificidad cultural y social de las expectativas de parto

- la escasez de estudios que, desde una aproximación cualitativa, exploren las expectativas de parto de las gestantes en España 
Por todo ello, en estos momentos resulta relevante una aproximación a las expectativas de parto de las gestantes desde un punto de vista cualitativo, debido a la adecuación de esa metodología para el estudio de sentimientos y percepciones. Por lo tanto, el objetivo de esta investigación es llevar a cabo una aproximación cualitativa al estudio de las expectativas de parto de una muestra de gestantes españolas. Asimismo, como objetivo secundario, se pretende realizar una comparación entre las expectativas de parto de mujeres primíparas y multíparas.

\section{MATERIAL Y MÉTODO}

El estudio se basó en una pregunta de respuesta abierta: “¿Cuáles son sus expectativas para el parto?, es decir, ¿qué desea o espera en su parto?". La pregunta formaba parte de un cuestionario más amplio, como se ha hecho en investigaciones previas $^{(7,18,20)}$. Los datos se recogieron entre junio de 2014 y enero de 2015 a gestantes de tercer trimestre de embarazo en el Hospital Universitario de la Ribera (Alzira, Valencia) que acudieron a puertas de urgencias de ginecología o a la consulta de control de bienestar fetal. Los criterios de inclusión fueron gestantes en el tercer trimestre de embarazo, mayores de edad, que se expresaran sin dificultad en cualquiera de las dos lenguas oficiales (castellano o valenciano) y que consintieran ser incluidas en el estudio. Se excluyeron del estudio a las gestantes que no cumplían dichos criterios. Se utilizó un muestreo de conveniencia, habitual en estudios de este

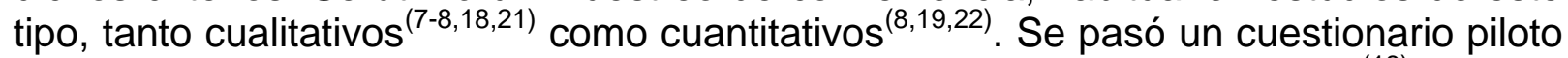
a un grupo reducido de gestantes (10 mujeres, al igual que Fenwick ${ }^{(18)}$, siendo habitual realizar el pilotaje entre $5^{(20)}$ y 20 mujeres $^{(7)}$ ). Finalmente, fue aprobado por el Servicio de Calidad del Hospital Universitario de la Ribera.

Se recogieron las siguientes variables de estudio:

- Edad expresada en años, variable categórica agrupada en 3 intervalos (de 18 a 25, de 26 a 35 y mayores de 35 años)

- Nivel de estudios, variable categórica con 4 categorías (sin estudios, estudios primarios, estudios secundarios y estudios universitarios)

- Fórmula obstétrica, categórica dicotómica (primípara o multípara)

- Pertenencia al área de salud de La Ribera, categórica dicotómica (pertenencia dentro o fuera del área de salud)

- Asistencia a las actividades de preparación al parto (categórica dicotómica sí/no)

- Expectativas de parto, pregunta de respuesta abierta sobre la que se realizó un análisis de contenido.

El análisis de contenido se llevó a cabo mediante un procedimiento similar al realizado en otros estudios cualitativos sobre esta temática ${ }^{(7-8,18,25)}$, y que consta de los siguientes pasos ${ }^{(24)}$ :

- Transcribir las respuestas y realizar una lectura de las mismas

- Identificar los temas comunes

- Codificar los datos para reducirlos a conceptos

- Estructurar los conceptos en categorías y subcategorías

Para aumentar la fiabilidad del análisis de los datos, fueron codificados por las 2 investigadoras de forma separada ${ }^{(5,25)}$ y, finalmente, fueron comparados, discutidos y reevaluados hasta crear códigos y categorías compartidas ${ }^{(24)}$. 
De forma similar a otros estudios ${ }^{(18,20)}$, las participantes dieron más de una respuesta a la pregunta de cuáles eran sus expectativas de parto. Se cuantificó el número total de respuestas de cada categoría en una hoja de cálculo Excel, junto con los datos socio-demográficos obtenidos. El tratamiento estadístico se llevó a cabo con el programa Statistical Package for the Social Sciences (SPSS) versión 17.0. Al ser todas las variables categóricas, la comparación de variables se realizó mediante la prueba de Chi-cuadrado para variables categóricas de Pearson (nivel de significación estadística $p<0.05$ para pruebas bilaterales). El estudio fue aprobado por el Comité de Ética de la Investigación-Comisión de Investigación del Hospital Universitario de la Ribera en junio de 2014. Ninguna gestante rechazó participar en el estudio y todas dieron su consentimiento informado.

\section{RESULTADOS}

Se recogió una muestra total de 213 gestantes que cumplían los criterios de inclusión, cuyos datos socio-demográficos se reflejan en la Tabla I. En total se obtuvieron 422 respuestas codificadas, incluyendo los casos en que la gestante manifestó que no tenía ninguna expectativa de parto $(n=11)$, tal como se observa en la Figura 1. Las respuestas fueron codificadas en 7 categorías (Figura 1) y 4 subcategorías.

Tabla I: Datos socio-demográficos de las gestantes de la muestra

\begin{tabular}{|cccc|}
\hline Variables & Valores & Frecuencia & Porcentaje \\
\hline \multirow{2}{*}{ Edad de la gestante } & $18-25$ & 17 & 8 \\
& $26-35$ & 146 & 68,5 \\
& $>35$ & 50 & 23,5 \\
\hline \multirow{2}{*}{ Fórmula obstétrica } & Primípara & 117 & 54,9 \\
& Multípara & 96 & 45,1 \\
\hline \multirow{2}{*}{ Área de salud } & La Ribera & 179 & 84 \\
& Otras & 34 & 16 \\
\hline \multirow{2}{*}{ Nivel de estudios } & Sin estudios & 1 & 0,5 \\
& Primarios & 63 & 29,6 \\
& Secundarios & 74 & 34,7 \\
& Universitarios & 75 & 35,2 \\
\hline \multirow{2}{*}{ Asistencia preparación al parto } & Sí & 131 & 61,5 \\
& No & 82 & 38,5 \\
\hline \multirow{2}{*}{ Asistencia charla tercer trimestre } & Sí & 147 & 69 \\
& No & 66 & 31 \\
\hline \multirow{2}{*}{} & & & \\
\hline
\end{tabular}


Figura 1: Expectativas de las gestantes de la muestra expresadas en porcentajes

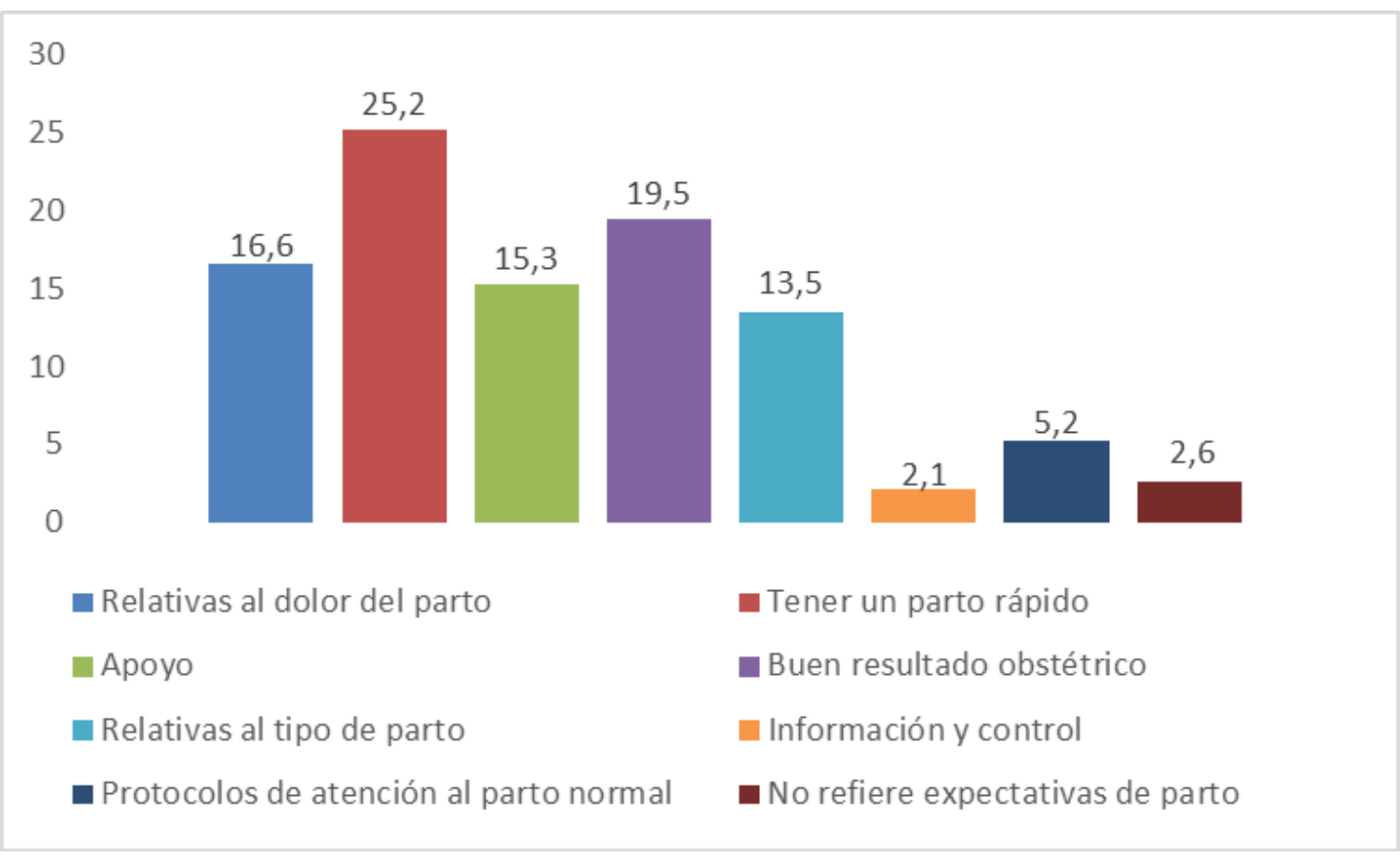

Dentro de la categoría "apoyo" se incluyeron las subcategorías "apoyo por parte de los profesionales sanitarios" ( $n=47)$ y "apoyo por parte de la persona elegida como acompañante" ( $n=18)$. La categoría "relativa al tipo de parto" incluyó las subcategorías "tener un parto normal/tener un parto vaginal" $(n=54)$ y "realización de una cesárea electiva" $(n=3)$.

En cuanto a la comparación en función de la variable fórmula obstétrica, el 57\% $(n=239)$ de las respuestas fueron aportadas por primíparas y el $43 \%(n=183)$ restante por multíparas (Tabla II). El análisis estadístico no arrojó relación estadísticamente significativa, en ninguna de las categorías, entre las expectativas de parto de las primíparas y multíparas de la muestra. A pesar de ello, los datos sí apuntan algunas tendencias, como por ejemplo la mayor frecuencia de expectativas relacionadas con los protocolos de atención al parto normal en las primíparas respecto de las multíparas.

Tabla II: Distribuciones de frecuencias y porcentajes de expectativas de parto en función de la variable paridad

\begin{tabular}{|lcccc|}
\hline \multicolumn{1}{c}{ Categorías } & \multicolumn{2}{c}{ Multíparas } & \multicolumn{2}{c|}{ Primíparas } \\
& Frecuencia & $\%$ & Frecuencia & $\%$ \\
\hline Relativas al dolor del parto & 29 & 15,8 & 41 & 17,1 \\
Tener un parto rápido & 49 & 26,9 & 57 & 23,8 \\
Apoyo & 29 & 15,8 & 36 & 15,2 \\
Buen resultado obstétrico & 32 & 17,6 & 50 & 20,9 \\
Relativas al tipo de parto & 28 & 15,2 & 29 & 12,2 \\
Información y control & 4 & 2,2 & 5 & 2,1 \\
Protocolos de atención al parto normal & 7 & 3,8 & 15 & 6,2 \\
No refiere expectativas de parto & 5 & 2,7 & 6 & 2,5 \\
Total & 183 & 100 & 239 & 100 \\
\hline
\end{tabular}


Por otro lado, en relación a la variable fórmula obstétrica y la asistencia a las actividades programadas para la educación maternal (preparación al parto y charla de tercer trimestre en hospital), sí que se ha encontrado una relación estadísticamente significativa (Tabla III). En este caso, la primiparidad se asocia con mayor asistencia a ambos tipos de actividades formativas prenatales.

\section{Tabla III: Análisis estadístico entre la variable fórmula obstétrica y las variables asistencia a preparación al parto y asistencia a la charla de tercer trimestre en el hospital}

\begin{tabular}{|lccccc|}
\multicolumn{1}{c}{ Variables } & Multiparas & Primiparas & $\begin{array}{c}\text { Chi } \\
\text { cuadrado }\end{array}$ & $\begin{array}{c}\text { Valor } \\
\text { de p }\end{array}$ & $\begin{array}{c}\text { V de } \\
\text { Cramer }\end{array}$ \\
\hline $\begin{array}{l}\text { Asistencia preparación } \\
\text { al parto }\end{array}$ & & & 30,3 & 0,0001 & 0,37 \\
Sí & 40 & 91 & & & \\
No & 57 & 25 & & & \\
Asistencia charla tercer & & & & & \\
trimestre en hospital & 55 & 93 & 13,1 & 0,0001 & 0,24 \\
Sí & 42 & 23 & & & \\
No & & & & & \\
\hline
\end{tabular}

\section{DISCUSIÓN}

La exploración de las expectativas de parto de una muestra de gestantes españolas ha permitido, por un lado, clasificar tales expectativas en 7 categorías y 4 subcategorías y, por otro lado, constatar la inexistencia de diferencias estadísticamente significativas entre las expectativas de primíparas y multíparas. Esta ausencia de diferencias difiere de los resultados de otros estudios, que sí detectan diferencias entre primíparas y multíparas, encontrando entre las multíparas una expectativa de parto más positiva ${ }^{(7,23)}$ y puntuaciones más bajas en la expectativa dolor $^{(19)}$. Existen diversas explicaciones posibles para esta diferencia, desde la adaptación de las expectativas actuales en base a la experiencia previa ${ }^{(3,7,23)}$ a la probabilidad de que las mujeres con una experiencia previa negativa no tengan otros embarazos $^{(23)}$. Con las debidas precauciones, nuestros resultados avalarían que se pueda trabajar indistintamente con muestras de solo multíparas ${ }^{(7,18)} 0$ solo primíparas $^{(8-9,20,25)}$. Según lo anterior, allí donde se detectan las diferencias, estas podrían no estar relacionadas con la paridad, sino con otros factores explicativos. Por ejemplo, Tarkka ${ }^{(6)}$ refiere que las mujeres con una actitud más positiva tienen más posibilidades de tener una experiencia de parto positiva. Según esto, podríamos decir que la actitud materna podría ser uno de esos factores explicativos.

A pesar de no haber encontrado significación estadística, hay no obstante alguna tendencia resaltable, como es, entre las primíparas, la mayor frecuencia de expectativas relacionadas con los protocolos de atención al parto normal (Tabla II). Este es precisamente un aspecto que se ha introducido recientemente en la asistencia al parto en el Hospital Universitario de La Ribera. Esta mayor frecuencia podría estar relacionada con la asistencia de las primíparas a las actividades formativas de preparación al parto (en las que se les informa de los protocolos introducidos recientemente), aspecto que sí presenta significación estadística en el presente estudio (Tabla III). 
Con respecto a la clasificación en 7 categorías, la expectativa con la frecuencia más elevada ha sido "tener un parto rápido" $(n=106)$. En el caso de los estudios cuantitativos, se expresa a través del ítem "I hope this labour will go smoothly, normally and fast" ("espero que este parto sea fluido, normal y rápido") del CEQ ${ }^{(19)}$ y de un ítem del ECBE, donde se expresa de forma negativa (tener un parto muy largo), apareciendo en un $66 \%$ de las gestantes encuestadas ${ }^{(9)}$. También se recoge en algunos estudios cualitativos ${ }^{(8,18)}$, siendo una de las expectativas más importantes en la investigación llevada a cabo por Gibbins ${ }^{(8)}$, al igual que en el presente estudio. Tener como expectativa un parto rápido o fácil se entiende como una expectativa irreal o idealizada, según Beaton ${ }^{(10)}$, y el punto de partida para tener una desilusión o decepción con la experiencia de parto subsiguiente.

La otra categoría principal obtenida por Gibbins ${ }^{(8)}$, el "dolor del parto", aparece en tercer lugar $(\mathrm{n}=70)$ en este caso. La recogen la mayoría de los estudios cualitativos ${ }^{(5,7-}$ $8,18,20,25)$, así como algunos cuantitativos que usan como herramienta el $\mathrm{CEQ}^{\left({ }^{(9)}\right.}$. En contrapartida, en otros estudios cuantitativos que utilizan ese mismo cuestionario no se recoge entre las expectativas mayores. Así, para Peñacoba ${ }^{(19)}$, el dolor del parto solo es importante para el $20 \%$ de las gestantes encuestadas y Zhang ${ }^{(22)}$ evidencia que el $68 \%$ de las gestantes deseaban adoptar métodos de alivio del dolor no farmacológicos, sin hacer referencia específica al dolor de parto como expectativa.

La seguridad y salud tanto de la madre como del bebé es la segunda expectativa en cuanto a su frecuencia $(\mathrm{n}=82)$ y se ha categorizado como "tener un buen resultado obstétrico". Se ha encontrado en la mayoría de los estudios cualitativos consultados $^{(5,7-8,18,20,25)}$, estando ausente en la mayor parte de estudios cuantitativos $^{(9,22)}$; el de Peñacoba ${ }^{(19)}$ constituye una excepción. El hecho de que aparezca de forma habitual en los estudios cualitativos y de forma minoritaria en los cuantitativos podría interpretarse como una insuficiencia en los cuestionarios a la hora de captar esta cuestión.

En referencia a la categoría "apoyo", que se ha dividido en dos subcategorías (apoyo por parte de los profesionales y apoyo por parte de la persona elegida como acompañante), los resultados indican que el apoyo por parte de los profesionales tiene un peso mucho mayor que el correspondiente a los acompañantes. Este resultado es consistente con otros estudios donde el apoyo por parte de los profesionales se perfila como el más significativo para mejorar la experiencia de la mujer durante el parto ${ }^{(4,6,15)}$, mientras que el apoyo del acompañante es también importante, pero no muestra el mismo efecto positivo ${ }^{(28)}$. Además, existen estudios que muestran una asociación entre apoyo profesional y corta duración del parto ${ }^{(28)}$, e incluso que el apoyo por parte de las matronas es capaz de motivar a buscar un futuro embarazo a aquellas mujeres que han tenido una mala experiencia durante el parto ${ }^{(7)}$. En diferentes aproximaciones cualitativas, las gestantes expresan sus miedos acerca de tres aspectos, la calidad del cuidado que podrían recibir por parte de los profesionales ${ }^{(25)}$; la posibilidad de ser mal tratadas por los mismos ${ }^{(7,9,20)}$; y la importancia de ser tratada de forma individualizada ${ }^{(7,18)}$.

Sin embargo, en los estudios cuantitativos, el apoyo de los acompañantes tiene un mayor peso que el apoyo por parte de los profesionales ${ }^{(19,22,29)}$. Esta discrepancia entre los resultados de estudios cualitativos y cuantitativos se podría explicar por la diferencia en el número de ítems que hacen referencia al apoyo por parte de los acompañantes y por parte de los profesionales en los cuestionarios. En este contexto queda patente el interés y utilidad de las metodologías cualitativas para el estudio de 
las expectativas de parto, ya que se evitan presunciones o teorías generadas de antemano $^{(24)}$.

Por su parte, respecto del apoyo de los profesionales, diferentes estudios ponen de relieve la importancia de la figura de la matrona y el poder que ejercen sobre las mujeres en el momento del parto ${ }^{(30)}$. Así, estas profesionales del parto suponen una gran fuente de apoyo físico, emocional y de información, además del rol técnico que se espera de ellas ${ }^{(5)}$. Las matronas deben usar ese poder de forma sensible e inteligente para conseguir el mayor beneficio para la mujer que está a su cargo, tomándose su tiempo para discutir las expectativas de las gestantes y asegurarse de su realismo y pertinencia ${ }^{(5)}$.

Las expectativas "relativas al tipo de parto" se subdividen en 2 categorías, aquellas gestantes que desean tener un parto "normal" o "vaginal" ( $n=54$, expresado con sus propias palabras, igual que ocurre en otros estudios ${ }^{(18)}$, donde se usan ambos términos de forma indistinta), y aquellas otras que desean que se les realice una cesárea electiva $(n=3)$. Este interés por tener un parto normal aparece en estudios cualitativos donde las gestantes están sensibilizadas hacia el parto humanizado (20-21) (ambos estudios realizados en Brasil, donde existe un importante movimiento para la humanización del parto). Además, es la expectativa más importante $(n=112)$, junto con la rapidez del parto, en el estudio realizado por Fenwick ${ }^{(18)}$ en Australia. En los estudios cuantitativos el interés por el tipo de parto se encuadra dentro del ítem "I hope this labour will go smoothly, normally and fast" (espero que este parto sea fluido, normal y rápido), constituyendo una de las expectativas mayores del estudio de Peñacoba en gestantes españolas ${ }^{(19)}$.

En el presente estudio se ha diferenciado entre la expectativa de tener un parto normal y todas aquellas englobadas dentro de los "protocolos de atención al parto normal". Estos últimos han tenido una frecuencia escasa, probablemente debido a su reciente introducción dentro del modelo asistencial. Sin embargo, en otros estudios es difícil diferenciar si las gestantes que expresan su deseo de tener un parto normal se refieren simplemente a un parto por vía vaginal (tal como lo interpreta Fenwick) ${ }^{(18)}$, 0 bien a aspectos relacionados con los protocolos de atención al parto normal. En este estudio se ha seguido la interpretación de Fenwick ${ }^{(18)}$.

Por último, la necesidad de "información y control" constituye el grupo de expectativas con la menor frecuencia $(n=9)$. En referencia a otros estudios, solo se ha encontrado en el realizado por Fenwick ${ }^{(18)}$, que lo plantea como una subcategoría dentro de la categoría "implicación y participación en el parto". En otras investigaciones cualitativas se hace referencia al control como confianza en el cuidado que les ofrecen los profesionales sanitarios ${ }^{(5)}$. Sin embargo, la sensación de control que proporciona la información se considera muy importante en algunos estudios, de cara a afrontar la experiencia de parto de forma positiva, incluso cuando el parto no se ajusta a las expectativas previas ${ }^{(8)}$. Por lo tanto, la información se perfila como un elemento clave para generar unas expectativas más precisas y específicas $^{(3,15)}$. Los profesionales sanitarios en general, y las matronas en particular, constituyen una excelente fuente de información, sobre todo en los países donde existe la oportunidad de asistir a clases de preparación al parto o educación maternal $^{(9,15,18)}$. Consecuentemente, los profesionales sanitarios deberían conocer las fuentes de información suplementarias que utilizan las gestantes (especialmente de aquellas mujeres que no asisten a educación maternal) y ofrecer fuentes de información reconocidas cuando sea necesario ${ }^{(5)}$. Debido al elevado interés de este 
tema, cabe plantearlo como futura línea de investigación, atendiendo, en primer lugar, a la exploración de las distintas fuentes de información que manejan las gestantes y, en segundo lugar, al origen de la información (contrastando la proporcionada por las matronas y otros profesionales sanitarios con otras posibles fuentes de información suplementarias).

\section{CONCLUSIONES}

El conocimiento de las expectativas de las gestantes es un aspecto crucial para poder ofrecer el mejor cuidado de salud posible en la atención al parto, adecuando la experiencia de parto de las gestantes a sus expectativas con el fin de lograr el mayor grado de satisfacción con el proceso de parto. En el actual momento de cambio de modelo asistencial resulta todavía más necesario conocer las expectativas de parto de las gestantes, ya que el modelo humanizado de atención al parto implica que la mujer tome decisiones sobre su cuidado e insiste en el concepto de comunicación entre usuarios y profesionales sanitarios.

La aproximación cualitativa al estudio de las expectativas de una muestra de gestantes españolas ha permitido clasificarlas en 7 categorías (parto rápido, relacionadas con el dolor, buen resultado obstétrico, apoyo, relacionadas con el tipo de parto, información y control, y relacionadas con los protocolos de atención al parto normal), sin diferencias estadísticamente significativas entre las expectativas manifestadas por las gestantes primíparas y multíparas.

Entre las expectativas más importantes destacan las relacionadas con tener un parto rápido, con un buen resultado obstétrico y sin experimentar dolor. Sin embargo, aquellas relacionadas con los protocolos de atención al parto normal tienen una presencia escasa, posiblemente debido a la reciente introducción de dicho modelo asistencial. Asimismo, resalta la importancia del apoyo prestado por los profesionales sanitarios, y en concreto la matrona, como actor principal para conseguir alinear expectativas y experiencias de parto de las gestantes. La matrona es el profesional más adecuado para proveer a las gestantes de una información de calidad sobre aspectos relacionados con el embarazo, el parto y la maternidad, colaborando de forma importante en la elaboración de expectativas realistas y en el empoderamiento de la gestante acerca de la toma de decisiones sobre las cuestiones que les afectan.

Se considera interesante como línea de investigación futura la exploración de las distintas fuentes de información que influyen en la generación de las expectativas de parto de las gestantes, así como el papel que desempeña la información que proporcionan los profesionales sanitarios dedicados a la atención al parto.

\section{REFERENCIAS}

1. Pinheiro BC, Bittar, CML. Expectativas, percepções e experiências sobre o parto normal: Relato de um grupo de mulheres. Fractal Revista de Psicologia. 2013;25(3):585-602. http://dx.doi.org/10.1590/S1984-02922013000300011

2. Soet JE, Brack GA, Dilorio C. Prevalence and predictors of women's experience of psychological trauma during childbirth. Birth. 2003;30:36-46.

3. Hauck Y, Fenwick J, Downie J, Butt J. The influence of childbirth expectations on western Australian women's perceptions of their birth experience. Midwifery. 2007;23,235-247. http://dx.doi.org/10.1016/j.midw.2006.02.002. 
4. Green JM, Baston HA. Feeling in control during labor: concepts, correlates, and consequences. Birth. 2003;30:235-247.

5. Martin DK, Bulmer SM, Pettker CM. Childbirth expectations and sources of information among low- and moderate- income nulliparous pregnant women. J Perinat Educ. 2013;22(2):103-112. http://dx.doi.org/10.1891/1058-1243.22.2.103

6. Tarkka MT, Paunonen M, Laippala P. Importance of the midwife in the first-time mother's experience of childbirth. Scand J Caring Sci. 2000;14(3):184-190.

7. Rilby L, Jansson S, Lindblom B, Mårtensson LB. A Qualitative Study of Women's Feelings About Future Childbirth: Dread and Delight. J Midwifery Womens Health. 2012;57(2):120-125. http://dx.doi.org/10.1111/j.1542-2011.2011.00113.x.

8. Gibbins J, Thomson AM. Women's expectations and experiences of childbirth. Midwifery. 2001;17(4):302-313.

9. Oweis A, Abushaikha L. Jordanian pregnant women's expectations of their first childbirth experience. Int $J$ Nurs Pract. 2004;10(6):264-271. http://dx.doi.org/10.1111/j.1440-172x.2004.00488.x.

10. Beaton J, Gupton A. Childbirth expectations: a qualitative analysis. Midwifery. 1990;6(3):133-139.

11. Christiaens W, Verhaeghe $M$, Bracke P. Childbirth expectations and experiences in Belgian and Dutch models of maternity care. $J$ Reprod Infant Psychol. 2008;26(4):309-322.

12. Ayers S, Pickering AD. Women's expectations and experience of birth. Psychol Health. 2005;20(1):79-92.

13. Sweeny K, Krizan Z. Sobering up: A quantitative review of temporal declines in expectations. Psychol Bull. 2013;139(3):702-724. http://dx.doi.org/10.1037/a0029951.

14. Gupton A, Beaton J, Sloan J, Bramadat I. The development of a scale to measure childbirth expectations. Can J Nurs Res. 1991;23(2):35-47.

15. Lally JE, Murtagh MJ, Macphail S, Thomson R. More in hope than expectation: A systematic review of women's expectations and experience of pain relief in labour. BMC Medicine. 2008;6:7. http://dx.doi.org/10.1186/1741-7015-6-7.

16. Ferreiro-Losada MT, Díaz-Sanisidro E, Martínez-Romero MD, Rial- Boubeta A, Varela-Mallou J, Clavería-Fontán A. Evaluación mediante grupos focales de las expectativas y percepciones de las mujeres durante el proceso de parto. Rev Calid Asist. 2013;28(5):291-299.

17. Goberna Tricas J, Palacio Tauste A, Banús Giménez MR, Linares Sancho S, Salas Casas D. Tecnología y humanización en asistencia al nacimiento. La percepción de las mujeres. Matronas Prof. 2008;9(1):5-10.

18. Fenwick J, Hauck Y, Downie J, Butt J. The childbirth expectations of a selfselected cohort of Western Australian women. Midwifery. 2005;21(1):23-35.

19. Peñacoba-Puente C, Carmona-Monge FJ, Marín-Morales D, Écija-Gallardo C. Evolution of childbirth expectations in Spanish pregnant women. Appl Nurs Res. 2016;29:59-63. http://dx.doi.org/10.1016/j.apnr.2015.05.017

20. Almeida N, Fleury EM. Expectativas de Gestantes sobre o Parto e suas Percepções acerca da Preparação para o Parto. Temas em Psicologia. 2016;24(2):681-693. http://dx.doi.org/10.9788/TP2016.2-15

21. Basso JF, Monticelli M. Expectativas de participação de gestantes e acompanhantes para o parto humanizado. Rev Lat Am Enfermagem. 2010;18(3):97105. http://dx.doi.org/10.1590/S0104-11692010000300014

22. Zhang $\mathrm{X}, \mathrm{Lu} \mathrm{H}$. Childbirth expectations and correlates at the final stage of pregnancy in Chinese expectant parents. International Journal of nursing sciences. 2014;(1):151-6. http://dx.doi.org/10.1016/j.ijnss.2014.05.019

23. Wijma K, Söderquist J, Wijma B. Posttraumatic stress disorder after childbirth: A cross sectional study. J Anxiety Disord. 1997;11(6):587-597. 
24. Graneheim UH, Lundman B. Qualitative content analysis in nursing research: concepts, procedures and measures to achieve trustworthiness. Nurse Educ Today. 2004;24(2):105-112.

25. Serçekus $P$, Okumus $H$. Fears associated with childbirth among nulliparous women in Turkey. Midwifery. 2009;25(2):155-162.

26. Biurrun-Garrido A, Goberna-Tricas J. La humanización del trabajo de parto: necesidad de definir el concepto. Revisión de la bibliografía. Matronas Prof. 2013;14(2):62-66 .

27. National Collaborating Centre for Women's and Children's Health. Intrapartum Care. Care of healthy women and their babies during childbirth. Clinical Guideline 190. Methods, evidence and recommendations; 2014. Disponible en: https://www.nice.org.uk/guidance/CG190 (último acceso 22 de noviembre 2016).

28. McGrath SK, Kennell JH. A randomized controlled trial of continuous labor support for middle-class couples: effect on cesarean delivery rates. Birth. 2008;35(2):92-97.

29. Udofia EA, Akwaowo CD. Pregnancy and after: What women want from their partners listening to women in Uyo, Nigeria. J Psychosom Obstet Gynaecol. 2012;33(3):112-119. http://dx.doi.org/10.3109/0167482X.2012.693551.

30. Anderson T. Feeling safe enough to let go: the relationship between a woman and her midwife during the second stage of labor. London: Palgrave Macmillan; 2000. 